\title{
Onset of Motion and Glassy Dynamics of a Moving Vortex Lattice
}

\author{
Guohong Li, Eva Y. Andrei, Xu Du, Z. L. Xiao*, P. Shuk* and M. Greenblatt \\ Department of Physics and Astronomy, Rutgers University, Piscataway, NJ 08855 USA \\ Department of Physics, Northern Illinois University, DeKalb, Illinois 60115, USA \\ "Department of Chemistry, Rutgers University, Piscataway, NJ 08855, USA
}

\begin{abstract}
We study the onset of motion of a vortex lattice in response to a driving current that is turned on suddenly. For sufficiently high currents we find that the average vortex velocity (obtained from the longitudinal voltage drop) grows smoothly with time and obeys a stretched exponential with an exponent $\beta \sim 0.7$. The response is governed by two time scales: a delay time $\left(t_{0}\right)$ before the appearance of a measurable voltage drop, and a rise time $(\tau)$ characterizing the evolution of voltage response. Both time scales increase with decreasing saturation voltage $V_{0}: \tau$ decreases exponentially, while $t_{0}$ as a power law. When the saturation velocity is too low the response slows down significantly and shows a step structure indicating plastic response.
\end{abstract}

\section{INTRODUCTION}

The structure of vortex lattices in type-II superconductors has been a topic of intense study over the past 50 years [1-3]. Our current understanding of its static properties can be summarized in terms of a global phase diagram containing a liquid and a variety of glassy phases separated by boundaries that are sensitive to material parameters including the amount and nature of imperfections, the extent of anisotropy, the coherence length and the London penetration length. One of the important advances in this field was the prediction that at low field and temperature and in the limit of clean samples the vortex lattice should form a quasi-ordered phase, the so-called Bragg glass [4,5]. This was shown to be a true thermodynamic phase characterized by the absence of topological defects, power law Bragg peaks and broken replica symmetry. The latter is expected to manifest itself as glassy dynamics [5-7] in experiments on moving vortex lattices. Static evidence for the existence of an ordered state consistent with the Bragg glass phase was thus far obtained through imaging experiments in both real and reciprocal space [8-10]. However little was done to probe the vortex motion in search of its putative glassy dynamics.

Most early studies on vortex motion employed transport measurements to obtain the vortex velocity from the steady-state voltage response to an applied current assuming that it is uniquely determined by the current amplitude. This assumption is reasonable for describing single vortex motion considering that vortices are overdamped objects whose response to a driving force is almost instantaneous. However experiments showing evidence of metastability, history effects and slow response times in weak pinning vortex lattices [11-13], demonstrated that the motion of a vortex lattice can differ significantly from that of a single vortex. Here we describe time-resolved experiments to study the onset of motion of a vortex lattice in response to an applied driving force. We find that the time evolution of the average vortex velocity when driven toward a high saturation 
velocity is described by a stretched exponential function, which is one of the salient properties of a glass $[14,15]$. The smooth evolution becomes significantly slower and even shows steps when driven to a low saturation velocity. A dynamic phase diagram is identified accordingly.

\section{EXPERIMENTAL}

In order to study the dynamic response of a driven vortex lattice, two questions have to be answered: What is the initial stationary state? How to measure the dynamic process?

There are various ways to prepare a vortex lattice. 1) Zero-field-cooling (ZFC), the sample is cooled through its superconducting transition temperature $T_{\mathrm{c}}$, and then a magnetic field is ramped up. 2) High-field-cooling (HFC), the sample is cooled in a very strong magnetic field, and then the field is ramped down. 3) Field-cooling (FC), the sample is cooled in the fixed field with the given level precisely. 4) Shaking, a small AC field is used to equilibrate the vortex lattice after ZFC or FC. 5) Driving, a driving current is used to help the equilibration. The FC state deserves special attention because as vortices form upon cooling through $T_{\mathrm{c}}$ their density is uniform and remains this way since the procedure does not involve introducing or removing vortices through the sample edges as is the case in the other methods.

Our experimental protocol is as follows. Starting from a freshly prepared FC state, we apply a current pulse and employ a time resolved measurement to monitor the evolution of the voltage induced by the vortex motion. This procedure allows monitoring the vortex lattice motion while it explores its configuration space under controlled experimental conditions.

Experiments were carried out on an un-doped single crystal of $2 \mathrm{H}-\mathrm{NbSe}_{2}$, with dimensions 4.41 $\mathrm{mm} \times 0.83 \mathrm{~mm} \times 6 \mu \mathrm{m}$, transition temperature and width of $T_{c}=7.2 \mathrm{~K}$ and $130 \mathrm{mK}$. The magnetic field was applied along the c-axis and the transport currents were in the a-b plane. Details of the experimental setup have been reported elsewhere [16].

\section{RESULTS AND DISCUSSION}

$2 \mathrm{H}-\mathrm{NbSe}_{2}$ is a typical weak pinning type-II superconductor. The static phase diagram of the sample was reported elsewhere [16]. SANS measurements on similar samples exhibit sharp Bragg peaks at low temperatures that are consistent with the formation of a Bragg glass $[9,10]$. In the following we focus on the dynamics of vortex lattices prepared by an FC process in $0.2 \mathrm{~T}$.

\subsection{Dynamic phase diagram}

The results on the evolution of the voltage response to an applied current are summarized in fig. 1. For a given temperature the saturation voltage $V_{0}$ increases linearly with current, $I$, as expected from free flux flow (fig. 1a). However, the time scale of the evolution slows down with decreasing $V_{0}$ (fig. 1b). As $V_{0}$ continues to decrease there is a transition in the behavior of $V(t)$ from fast and smooth to slow and stepwise. In order to capture the main features of $V(t)$ we define a response time, $\tau_{80}$, as the time when $80 \%$ of $V_{0}$ is reached. Fig.1c is a contour plot of $\tau_{80}$ in the $V_{0}-T$ plane. Due to the presence of a surface barrier [17], the applied current is not proportional to the bulk current density $J$. But since $J$, rather than the total current, determines the driving force on individual vortices, $f_{d} \sim J$, the applied current is not a reliable measure of the force. Instead we use $V_{0}$ as a measure of the driving force:

$$
V_{0} \propto\left(f_{d}-f_{p}\right) / \eta
$$

where $f_{p} \sim J_{p}$ is the pinning force, $\eta$ the viscosity and $J_{p}$ is the critical current density. Thus one can treat the vertical axis of fig. $1 \mathrm{c}$ as a force axis. 
One of the outstanding features of fig. $1 \mathrm{c}$ is the existence of a bubble (defined by the squares) within which $\tau_{80}$ is several orders of magnitude longer. In the bubble regime the response breaks up into long time steps during which only a fraction of the vortices are moving - a clear signature of plastic response. The arrow in the figure points to the same $V_{0}$ as that in fig. $1 \mathrm{~b}$ showing the definition of the bubble boundary as the locus for the appearance of stepwise response. Another feature is that $\tau_{80}$ depends weakly on temperature below $\sim 5.70 \mathrm{~K}$ (dashed line in fig. 1c) but decreases sharply above it.
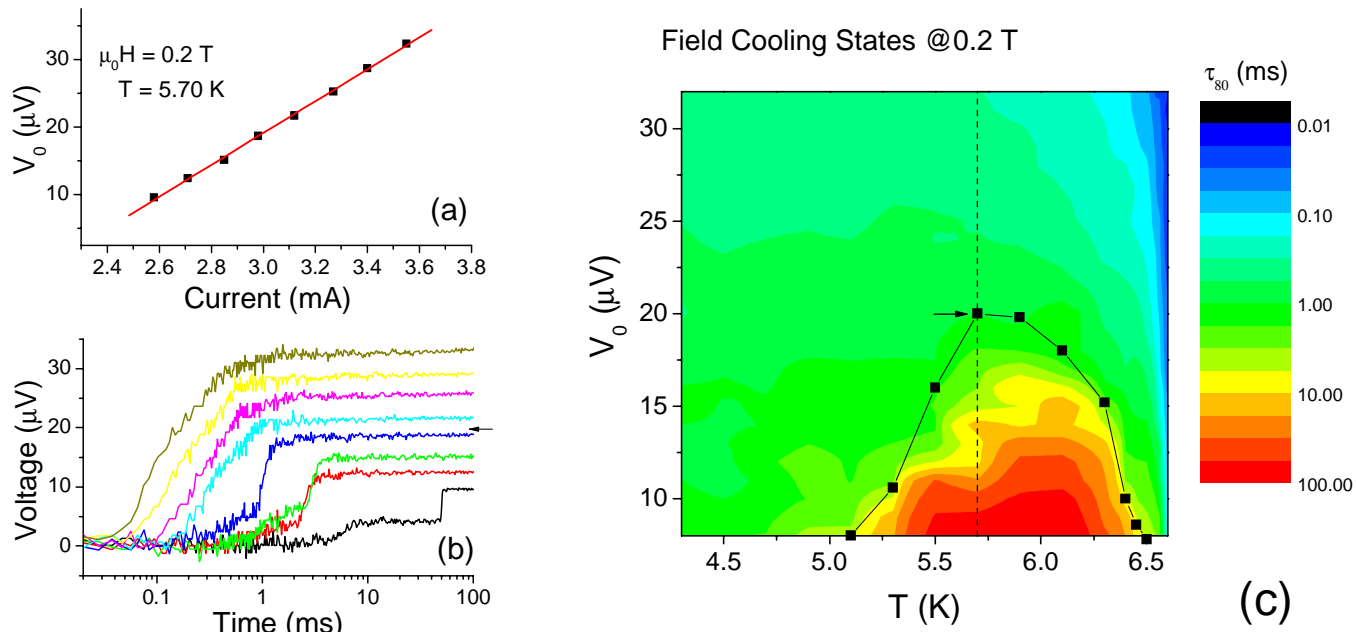

Figure 1. a) Saturation voltage $V_{0}$ versus applied current level. The solid line is a fit to free flux flow. b) Time dependence of voltage for different $V_{0}$. The response is smooth at high levels but step-like at low levels. The arrow shows the boundary separating the regimes of elastic and plastic flow. c) The contour plot of response times (see text).

\subsection{Evolution toward high velocity}

In fig. 2 we show the time dependence of the response in the high velocity regime where it is smooth (outside the bubble of fig. 1c). In this regime the data is well described by a stretched exponential:

$$
V(t)=V_{0}\left(1-e^{-\left(\frac{t-t_{0}}{\tau}\right)^{\beta}}\right), \quad 0<\beta<1
$$

where $V_{0}$ is the saturation voltage, $t_{0}$ is a waiting time before any voltage appears, $\tau$ is the rise time and $\beta=0.7$. In the same figure we also show that a fit to a simple exponential decay cannot follow the data over the entire range. Similarly a simple logarithmic fit, while adequate for short times must deviate from the data at longer times. The stretched exponential response observed here is one of the hallmarks of glassy behavior [14, 15]. It arises in the presence of many competing response times with the exponent typically characterizing the distribution of these times. The stretched exponential response inevitably arises when the dynamics of competing mechanisms is hierarchically constrained [15]. 


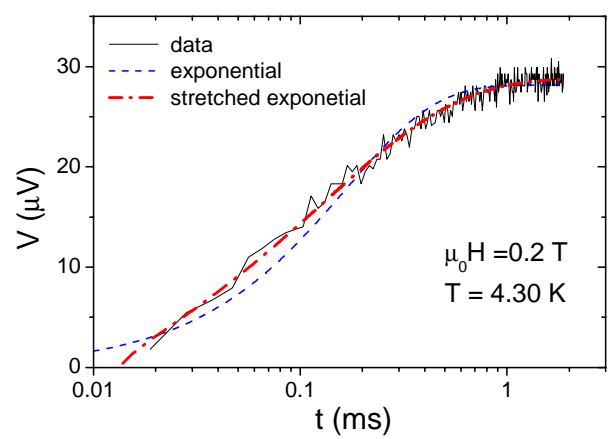

Figure 2. Time evolution of vortex response to an applied current pulse. The fits show that the response is best described by a stretched exponential function (equation 2 in the text) with $\beta=0.7$.

In Fig. 3 we show the temperature dependence of the fitting parameters $\tau$, $t_{0}$, and $\beta$ obtained by fitting the data for $V(t)$ to the stretched exponential function in equation 2 over the experimental temperature range. The upper panels describing the low temperature data are almost identical indicating weak temperature dependence consistent with the contour plot of Fig 1c. The average value of the exponent is found to be $\beta \sim 0.7$, with a scatter that decreases significantly at higher velocities.
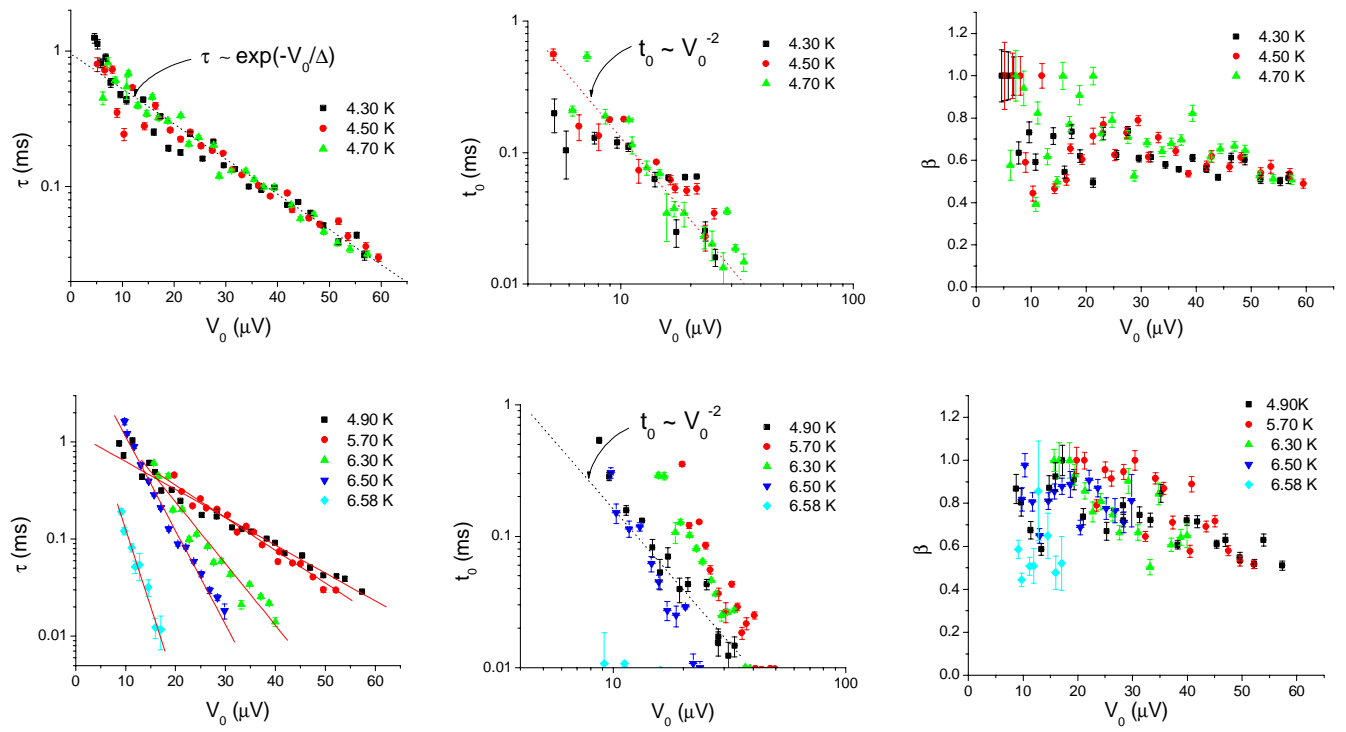

Figure 3. Fitting parameters as a function of saturation voltage obtained by fitting the voltage response with a stretched exponential at low temperatures (upper panels) and high temperatures (lower panels). The solid lines are fits to an exponential and the dotted lines are guides to the eye.

We note that the relaxation time $\tau$ decreases exponentially with $V_{0}$ while the delay time $t_{0}$ is better described by a power law $t_{0}\left(V_{0}\right) \sim V_{0}^{-\alpha}$. The power law dependence suggests a divergence in the delay time at the depinning threshold, $V_{0} \sim 0$. In the lower panels of Fig. 3 we note the onset of temperature dependence of the fitting parameters for $T>5.7 \mathrm{~K}$, however the exponential dependence of $\tau\left(V_{0}\right)$ persists at all temperatures. In fig. 4 we plot the temperature dependence of the exponent $\alpha$ and of $t_{0}$ at $V_{0}=10 \mu \mathrm{V}$. We note that the power increases almost linearly with temperature. 

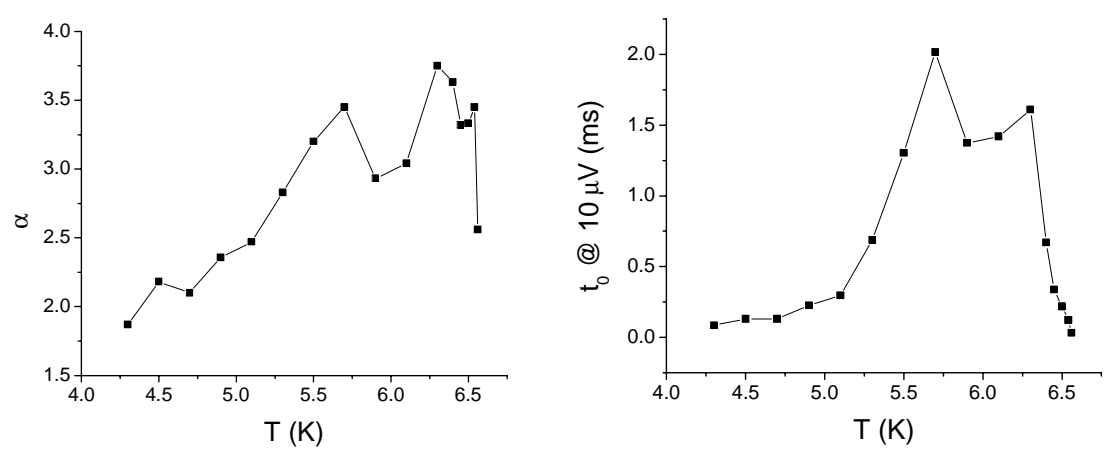

Figure 4. (left) Temperature dependence of the power $\alpha$ in $t_{0} \sim V_{0}^{-\alpha}$. (right) Temperature dependence of $t_{0}$ at $10 \mu \mathrm{V}$.

\subsection{Discussion}

When the vortex lattice moves in response to an applied current pulse it undergoes a transition from static to moving state and since the moving state is more ordered than the stationary one [18,19], the finite response time can be attributed to the time to heal lattice defects. If we assume that healing proceeds by an activated process with barrier $U\left(V_{0}, T\right)[16]$ the time scale of the defect healing rate would be given by $\tau \sim \exp (U / T)$. For a driven lattice the effect of the applied current on the barrier can be estimated using the Anderson Kim model [20] $U(J) \sim U_{c}\left(1-J / J_{c}\right)$ where $U_{c}$ is the static barrier due to pinning. Using equation 1 we find $J=V_{0} / \rho_{f f} l+J_{c}$, where $\rho_{f f}=\rho_{n} H / H_{c 2}$ is the free flux flow resistivity and $l$ the distance between voltage leads. Thus we can write

$$
\tau\left(V_{0}\right)=\tau_{0} e^{-\not N_{0} / T}
$$

with $\gamma=U_{c} T / \rho_{f f} l J_{\mathrm{c}}$ and $\tau_{0} \sim N_{0}(T)$ where $N_{o}(T) \sim e^{\alpha T}$ is the number of defects in the initial lattice [16].
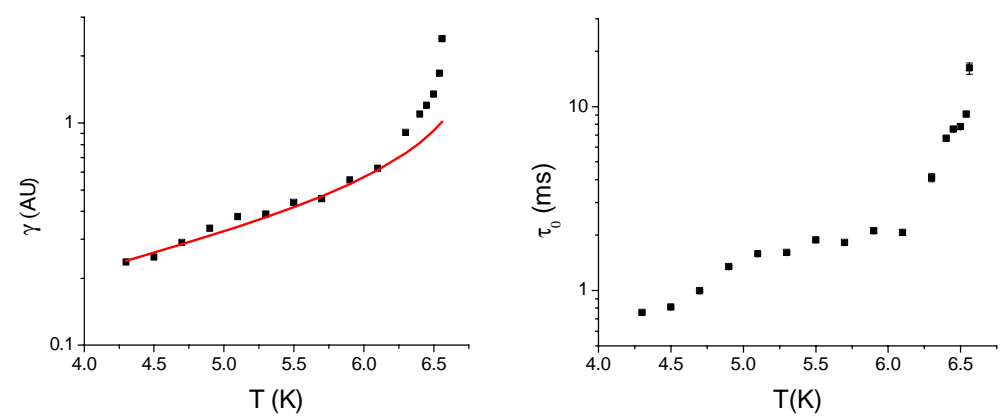

Figure 5. Temperature dependence of $\gamma$ and $\tau_{0}$ obtained by fitting to equation (3). The solid line in the left panel describes the temperature dependence of $\gamma$ calculated by assuming an activation process for healing defects and an Anderson-Kim model to describe the effect of the driving force on the barrier.

The values of $\mathcal{X} T)$ and $\tau_{0}(T)$ obtained from fitting the data are plotted in figure 5. In the left panel we compare the temperature dependence of the measured $\chi(T)$ to that obtained from a collective pinning [2] model $U_{c} T / \rho_{f f} l J_{\mathrm{c}} \sim\left(T_{c}-T\right)^{-1 / 2}$ (solid curve) where $T_{c}=6.9 \mathrm{~K}$ is the critical temperature of 
the sample at $0.2 \mathrm{~T}$. We note that for $T<6 \mathrm{~K}$ this model captures the temperature dependence $\chi T$ ). At higher temperatures the deviation from this model suggests that a new mechanism, such as injection of defects through sample edges, further slows down the healing process.

\section{SUMMARY}

We have studied the dynamic evolution of a vortex lattice from stationary to moving state in response to an applied current pulse. We find that the time evolution of the response follows a stretched exponential with exponent $\beta \sim 0.7$, as expected of a glassy system The response is characterized by two time scales, a delay time $t_{0}$ and a characteristic response time $\tau$. Both time scales depend on the saturation voltage, with $t_{0}\left(V_{0}\right) \sim V_{0}^{-\alpha}, \alpha \sim 1.8-3.8$ and $\tau\left(V_{0}\right)=\tau_{0} e^{-\gamma_{0} / T}, \gamma(T) \sim\left(T-T_{\mathrm{c}}\right)^{-1 / 2}$ for sufficiently large values of $V_{0}$ and low temperatures. When the vortex lattice is driven towards a low $V_{0}$, plastic processes set in leading to the appearance of steps and non-monotonous response. We attribute the time evolution of the response to a process of defect healing in the vortex lattice and show that the temperature dependence of the response is consistent with an activation process over a barrier that is reduced in the presence of the applied current according to the Anderson Kim model.

\section{Acknowledgments}

Work supported by NSF-DMR-0456473 and DOE-DE-FG02-99ER45742.

\section{References}

[1] A. M. Campbell and J. E. Evetts, Adv. Phys. 21, 199 (1972).

[2] G. Blatter et. al, Rev. Mod. Phys. 66, 1125 (1994).

[3] Giamarchi and S. Bhattacharya, in High Magnetic Fields. Applications in Condensed Matter Physics and Spectroscopy, edited by C. Berthier, L. P. Levy, and G. Martinez (Springer-Verlag, Berlin, 2001) p.314.

[4] T. Nattermann, Phys. Rev. Lett. 64, 2454 (1990)

[5] T. Giamarchi and P. Le Doussal, Phys. Rev. Lett. 72, 1530 (1994); T. Giamarchi and P. Le Doussal, Phys. Rev. B 52, 1242 (1995).

[6] M. Nicodemi and H. J . Jensen, Phys. Rev. B 65, 144517 (2002).

[7] C. J. Olson et. al, Phys. Rev. B 67, 184523 (2003).

[8] H. F. Hess et. al, Phys. Rev. Lett. 62, 214 (1989).

[9] R. Cubitt et al., Nature (London) 365, 407 (1993)

[10] T. Klein et al., Nature (London) 413, 404 (2001).

[11] W. Henderson et. al, Phys. Rev. Lett. 77, 2077 (1996).

[12] J. A. Frendrich et. al, Phys. Rev. Lett. 77, 2073 (1996).

[13] B. Sas et. al, Phys. Rev. B 61, 9118 (2000).

[14] J.-P. Bouchaud, cond-matt/0408617.

[15] R. G. Palmer et. al, Phys. Rev. Lett. 53, 958 (1984).

[16] G. Li et. al, Phys. Rev. Lett. (submitted).

[17] Z. L. Xiao et. al, Phys. Rev. B 65, 094511 (2002).

[18] U. Yaron et. al, Phys. Rev. Lett. 73, 2748 (1994).

[19] D. W. Braun et. al, Phys. Rev. Lett. 76, 831 (1996).

[20] P. W. Anderson and Y. B. Kim, Rev. Mod. Phys. 1, 39 (1964) 